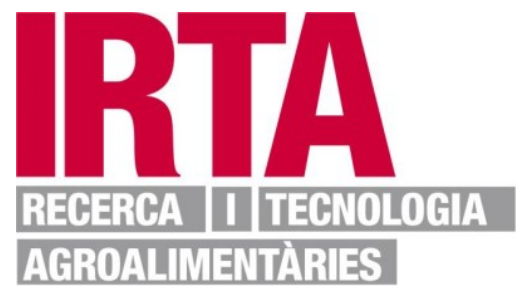

This document is a postprint version of an article published in Food Microbiology@ Elsevier after peer review. To access the final edited and published work see https://doi.org/10.1016/j.fm.2020.103649

Document downloaded from: 


\section{Quantifying the bioprotective effect of Lactobacillus sakei CTC494 against Listeria monocytogenes on vacuum packaged hot-smoked sea bream}

Authors: Araceli Bolívar ${ }^{*}$, Jean Carlos Correia Peres Costa ${ }^{1}$, Guiomar D. PosadaIzquierdo $^{1}$, Sara Bover-Cid ${ }^{2}$, Gonzalo Zurera ${ }^{1}$, Fernando Pérez-Rodríguez $^{1}$

${ }^{1}$ Department of Food Science and Technology, Faculty of Veterinary, Agrifood Campus of International Excellence (ceiA3), University of Cordoba, 14014, Córdoba, Spain.

${ }^{2}$ IRTA-Food Safety Programme, Finca Camps i Armet s/n, 17121, Monells, Girona, Spain

*Corresponding author at: Department of Food Science and Technology, University of Cordoba, Campus de Rabanales s/n. Edif. Darwin_anexo (C1) Crta. Madrid-Cádiz Km 396A, 14014, Córdoba, Spain.

E-mail addresses: $\underline{\mathrm{t} 12 \mathrm{bocaa} @ u c o . e s}$ (A. Bolívar)

Keywords: biopreservation; ready-to-eat fish products; food safety; microbial interaction model; predictive microbiology, bacteriocin producing bacteria

\section{ABSTRACT}


In this study, the bioprotective potential of Lactobacillus sakei CTC494 against Listeria monocytogenes CTC1034 was evaluated on vacuum-packed hot-smoked sea bream at 5 ${ }^{\circ} \mathrm{C}$ and dynamic temperatures ranging from 3 to $12{ }^{\circ} \mathrm{C}$. The capacity of three microbial competition interaction models to describe the inhibitory effect of $L$. sakei CTC494 on $L$. monocytogenes was assessed based on the Jameson effect and Lotka-Volterra approaches. A sensory analysis was performed to evaluate the spoiling capacity of $L$. sakei CTC494 on the smoked fish product at $5{ }^{\circ} \mathrm{C}$. Based on the sensory results, the bioprotection strategy against the pathogen was established by inoculating the product at a 1:2 ratio (pathogen:bioprotector, $\log \mathrm{CFU} / \mathrm{g}$ ). The kinetic growth parameters of both microorganisms were estimated in mono-culture at constant storage $\left(5{ }^{\circ} \mathrm{C}\right)$. In addition, the inhibition function parameters of the tested interaction models were estimated in coculture at constant and dynamic temperature storage using as input the mono-culture kinetic parameters. The growth potential $(\delta \log )$ of $L$. monocytogenes, in mono-culture, was $3.5 \log$ on smoked sea bream during the experimental period (20 days). In co-culture, L. sakei CTC494 significantly reduced the capability of L. monocytogenes to grow, although its effectiveness was temperature dependent. The LAB strain limited the growth of the pathogen under storage at $5^{\circ} \mathrm{C}(<1 \log$ increase $)$ and at dynamic profile $2(<2 \log$ increase). Besides, under storage at dynamic profile 1, the growth of L. monocytogenes was inhibited (<0.5 log increase). These results confirmed the efficacy of L. sakei CTC494 for controlling the pathogen growth on the studied fish product. The LotkaVolterra competition model showed slightly better fit to the observed L. monocytogenes growth response than the Jameson-based models according to the statistical performance. The proposed modelling approach could support the assessment and establishment of bioprotective culture-based strategies aimed at reducing the risk of listeriosis linked to the consumption of RTE hot-smoked sea bream. 
1 1. INTRODUCTION

2 Smoked fish products are sold as ready-to-eat (RTE) foods characterized by a relatively

3 long refrigerated shelf-life when packaged under vacuum (Hwang, 2007). These seafood

4 commodities are popular, but they are also considered among the top risk foodstuffs since

5 they can be contaminated with foodborne pathogens and no cooking is applied before

6 consumption (Ghanbari et al., 2013). At present, the microbiological concerns in the EU

7 associated with extended shelf-life refrigerated RTE foods are focused on psychrotrophic

8 foodborne pathogens such as Listeria monocytogenes (EFSA BIOHAZ Panel, 2018).

9 Biopreservation, also called bioprotection, is a biocontrol approach to enhance product 10 safety and shelf-life using microorganisms selected for their antimicrobial properties, so 11 called protective cultures (Leroi et al., 2015). Lactic-acid bacteria (LAB) are considered a new generation of food additives and the basis of food biopreservation (Said et al., 2019). Protective cultures are considered by the regulatory agencies as 'new' food additives, meaning that they require market authorization for their technological use in

15 foods. However, most LAB are Generally Recognized as Safe (GRAS) and many LAB 16 species (including Lactobacillus sakei) have been granted by EFSA with the Qualified 17 Presumption of Safety (QPS) status (EFSA, 2018). In the EU, microorganisms with the latter food-grade standard do not need to undergo a further safety assessment other than to provide evidence of efficacy and to satisfy the specified qualifications, if applicable,

20 for its market approval. Two recent studies have proved that the antilisterial sakacin K21 producing Lactobacillus sakei strain CTC494 (from meat origin) is effective to inhibit $L$. monocytogenes in filleted sea bream and cold-smoked salmon under refrigerated storage (Aymerich et al., 2019; Costa et al., 2019). Nevertheless, the inhibitory capacity of this bioprotective LAB strain has not been tested in other fish products where the differences in product's characteristics and formulations might either favor its inhibition thanks to 
26 the antimicrobial hurdle combinations (Leistner, 2000) or hinder the ability of the strain

27 to inhibit L. monocytogenes (Tahiri et al., 2009; Vasilopoulos et al., 2010).

28 Quantifying microbial interaction in food can be highly complex and often overlooked in predictive microbiology studies (Powell et al., 2004). Most of the competitive growth 30 models available in literature are based on two approaches: one based on the Jameson 31 effect phenomenon (i.e. nutrient competition) (Jameson, 1962) and the other using the 32 general Lotka-Volterra competition model (i.e. predator-prey model) (Powell et al., 2004; Valenti et al., 2013). Both mathematical models represent a simultaneous deceleration of bacterial populations. The inhibition of L. monocytogenes by endogenous LAB usually responsible for spoilage has been studied and modelled in minimally processed fish 36 products (Mejlholm et al., 2015; Mejlholm and Dalgaard, 2015, 2007). In this regard, 37 most of the published microbial interaction models aim at describing competition between background microbiota and microbial pathogens, rather than to characterize the performance of bioprotective bacteria with specific antagonistic activities, that are 40 normally added at higher levels than the natural background (spoilage) microbiota (Cornu 41 et al., 2011). To the best author's knowledge, studies having quantified the bioprotective 42 effect of bacteriocin-producing LAB cultures through the development and 43 implementation of predictive models are scarce. The first attempt to model the inhibitory 44 effect of a bacteriocinogenic LAB strain against Listeria in fish was made by Costa et al. 45 (2019), in which model parameters were derived from experiments in a fish-based broth 46 and then validated on fresh filleted sea bream.

47 The objective of this study was (i) to evaluate the bioprotective potential of L. sakei 48 CTC494 against L. monocytogenes on hot-smoked sea bream under constant and dynamic storage temperature conditions and (ii) to evaluate the capacity of three microbial 
50 interaction models based on the Jameson effect and Lotka-Volterra approaches to

1

2 3 4

5

6

7

8

9

10

11

12

13

14

15

16

17

18

19

20

21

22

23

24

25

26

27

28

29

30

31

32

33

34

35

36

37

38

39

40

41

42

43

44

45

46

47

48

49

50

51

52

53

54

55

56

57

58

59

60

61

62

63

64

65

51 describe the inhibitory effect of L. sakei CTC494 on L. monocytogenes.

52 


\subsection{Bacterial strains}

55

56

57

The selected bacterial strains used in this work were the bioprotective culture L. sakei CTC494 and the target pathogen L. monocytogenes CTC1034. This pathogenic strain was used in a previous work as a reference strain to study the antagonism of bacteriocinproducing LAB, including L. sakei CTC494 (Garriga et al., 2002). Both microorganisms were stored at $-80{ }^{\circ} \mathrm{C}$ in the appropriate culture broth with $20 \%(\mathrm{v} / \mathrm{v})$ glycerol. Before inoculation, a fresh culture was prepared for each strain and a well-isolated colony was used to perform two consecutive $24 \mathrm{~h}$-subcultures, which were grown in de Man, Rogosa and Sharpe broth (MRS, Oxoid, UK) for L. sakei at $30{ }^{\circ} \mathrm{C}$ with $10 \% \mathrm{CO}_{2}$ and Brain Heart Infusion (BHI, Oxoid, UK) for L. monocytogenes at $37^{\circ} \mathrm{C}$. This resulted in a cell density of $c a .10^{8} \mathrm{CFU} / \mathrm{mL}$ and $10^{9} \mathrm{CFU} / \mathrm{mL}$ for L. sakei and L. monocytogenes, respectively.

\subsection{Preparation of hot-smoked sea bream}

Gilthead sea bream fishes (Sparus aurata) from marine aquaculture were collected and processed by the Andalusian Aquaculture Technology Centre (CTAQUA, Cádiz, Spain) following an industrial hot smoking process. First, fish samples were manually scaled, gutted, filleted and bled in ice-water. Then, fillets were brined in a $\mathrm{NaCl} /$ sugar solution (ratio 3:1) for 2 hours. After that, fish fillets were removed from the brine solution, washed with water and introduced in a smoking oven (till the fish core temperature reached $65^{\circ} \mathrm{C}$ during $30 \mathrm{~min}$ ). After cooling at room temperature for $15 \mathrm{~min}$, smoked fillets were vacuum-packed and transferred to the laboratory the day after processing in polystyrene boxes under cold conditions.

\subsection{Sensory assessment}


76 A sensory analysis was carried out to evaluate the impact of the bioprotective culture on

77 the organoleptic characteristics of hot-smoked sea bream during refrigerated storage based on an acceptance test. For that, $10 \mathrm{~g}$-fish portions were surface inoculated with the L. sakei CTC494 as described below (section 2.4) at three initial cell densities (ca. 2 log $\mathrm{CFU} / \mathrm{g}, 4 \log \mathrm{CFU} / \mathrm{g}$ and $6 \log \mathrm{CFU} / \mathrm{g}$ ) and stored at $5 \pm 0.5^{\circ} \mathrm{C}$ under vacuum packaging conditions for 18 days. A control batch was prepared without inoculating bacteria.

The sensory evaluation was performed based on the work by Yanar et al. (2006), who evaluated the shelf-life of hot smoked tilapia stored at $4{ }^{\circ} \mathrm{C}$. For that, 5 panelists were trained according to the standard EN ISO 8586:2012 method. The descriptors were generated by open discussion and consensus in a previous session using fish samples stored under the same experimental condition applied in this study. The descriptors retained were general appearance (score of the overall appearance), intensity of odour (score of the overall odour) and texture to the touch. The assessors scored control and inoculated samples for the appearance, odour and texture characteristics using a 9-point hedonic scale. A score of 7-9 denoted "very good" quality, a score of 4.0-6.9 "good"' 91 quality, and a score of 1.0-3.9 indicated "unacceptable" quality. The sensory assessment was performed on the storage days 4, 7, 11, 14 and 18. At each evaluation point, one fish portion from each assayed condition was individually served under white light on conventional petri dishes, labeled with three-digit random numbers.

95 The obtained sensory scores for each attribute were plotted against time and sensory deterioration rates were obtained by fitting a linear model. Deterioration rates (slopes) for inoculated and control samples were statistically compared by performing t-test $(\mathrm{p} \leq 0.05)$ using the statistical software package SPSS 25.0 (Chicago, Illinois, USA).

\subsection{Challenge tests}


100 In the first trial, the growth of mono-cultured and co-cultured L. sakei CTC494 and $L$. 101 monocytogenes CTC1034 on smoked sea bream was evaluated under constant 102 refrigerated storage $\left(5 \pm 0.5^{\circ} \mathrm{C}\right)$ for 20 days. Four batches were prepared: (1) non103 inoculated product (control); (2) inoculated with ca. 2 log CFU/g L. monocytogenes; (3) 104 inoculated with ca. $4 \log \mathrm{CFU} / \mathrm{g}$ L. sakei and (4) inoculated with a mixture of $L$. 105 monocytogenes and L. sakei at a ratio 1:2 (i.e. $2 \log$ CFU/g L. monocytogenes and $4 \log$ 106 CFU/g L. sakei). The inoculation ratio pathogen:bioprotector used in batch 4 was selected 107 based on the results previously obtained from the sensory analysis (section 2.3).

108 Prior to inoculation, smoked fish fillets were cut into portions of $c a .5 \mathrm{~cm}^{2}$ and $10 \mathrm{~g}$ using 109 a sterile scalpel. This fish portion was considered the analytical fish sample. The caudal 110 region of the fish fillet was discarded from the analysis to avoid experimental variability.

111 Smoked sea bream portions were surface inoculated on the flesh side at $1 \%(\mathrm{v} / \mathrm{w})$ from 112 the appropriate decimal dilution using a L-shaped sterile spreader. For experiments in co113 culture (batch 4), the product was first inoculated with the pathogen and left for $10 \mathrm{~min}$ 114 in the safety cabinet to allow cell attachment. The LAB strain was then spread, followed 115 by a cell attachment period as the pathogen case. This inoculation procedure (Aymerich 116 et al., 2019) was used to mimic a post-processing contamination scenario in which the 117 fish product is contaminated by the pathogen after the hot-smoking process and then the 118 bioprotective culture is applied. After inoculation, fish portions were individually 119 vacuum-packed in polyamide-polyethylene plastic bags (Sacoliva, Barcelona, Spain) and 120 stored as described above.

121 In the second trial (approx. 2 months later) with a new batch of hot-smoked sea bream, the bioprotective effect of L. sakei CTC494 on L. monocytogenes was evaluated in co-

123 culture at two different dynamic temperature profiles, which were designed to simulate 124 the fluctuating conditions of the cold-chain distribution and storage of smoked fish 
125 products in Spain. Profile 1 had a mean temperature of $6.3^{\circ} \mathrm{C}$ and range between 3.6 to

$126 \quad 12.8^{\circ} \mathrm{C}$ while profile 2 had $7.6^{\circ} \mathrm{C}$ of mean temperature and range between 3.3 to $11.8^{\circ} \mathrm{C}$.

127 Microorganisms were co-inoculated on the sample's surface and vacuum packaged as

128 described before for batch 4. An additional non-inoculated (control) batch was also

129 studied.

130 The challenge tests were conducted in Hot-Cold incubators containing programmable

131 time-temperature profiles (Selecta, Barcelona, Spain). Incubation temperatures were

132 measured continuously throughout the experiments by data loggers (MicroLite, Fourier

133 Technologies, Israel). For experiments, 2 samples (duplicate) were analysed for

134 microbiological and/or physicochemical determinations at different sampling points. The

135 experiments were repeated twice in different days.

\subsection{Microbiological analyses}

137 Fish portions of $10 \mathrm{~g}$ were aseptically transferred into a sterile stomacher bag and 138 homogenized for $60 \mathrm{~s}$ (masticator, IUL Instruments, Barcelona, Spain) with $0.1 \%$ sterile 139 peptone water (Oxoid, UK).

140 For non-inoculated (control) samples, the growth of aerobic mesophilic viable count 141 (MVC), endogenous LAB counts and Listeria investigation (presence/absence) was 142 evaluated during storage at the tested temperatures. MVC counts were determined by 143 pour plating decimal dilutions from homogenized samples in Plate Count Agar (PCA, 144 Oxoid) incubated $48 \mathrm{~h}$ at $37{ }^{\circ} \mathrm{C}$. For LAB enumeration, de Man, Rogosa, Sharpe agar 145 (MRS, Oxoid) was used, which was incubated $48 \mathrm{~h}$ at $33{ }^{\circ} \mathrm{C}$ with $10 \% \mathrm{CO}_{2}$. The 146 presence/absence of L. monocytogenes was carried out following the method EN ISO $147 \quad 11290-1$. 
148 For inoculated samples, the enumeration of L. monocytogenes was carried out using 149 Oxford agar (Oxoid) containing Oxford selective supplement (SR0140, Oxoid). Counts 150 were obtained after incubating the plates for $24-48 \mathrm{~h}$ at $37^{\circ} \mathrm{C}$. LAB were enumerated as 151 for non-inoculated samples.

\subsection{Physicochemical analyses}

153 The physiochemical characteristics of hot-smoked sea bream (i.e. $\mathrm{pH}$ and $\mathrm{a}_{\mathrm{w}}$ ) were 154 determined by analysis of 2 fish portions (10 g each). $\mathrm{pH}$ measurement was performed 155 for samples from batch 1 (non-inoculated) and batch 3 (inoculated with $c a .4 \log \mathrm{CFU} / \mathrm{g}$ 156 L. sakei) on the days of the sensory analysis (section 2.3) using the pH meter Edge HI2020 157 (HI11310 electrode, Hanna Instruments, USA) by homogenizing the fish portion with 158 distilled water at ratio 1:1.

159 The determination of $\mathrm{a}_{\mathrm{w}}$ was performed with the AquaLab 4TE $\mathrm{a}_{\mathrm{w}}$ meter (Decagon 160 Devices Inc., WA, USA) for non-inoculated samples at the start of the challenge tests.

\subsection{Modelling microbial interaction}

162 The inhibition of L. monocytogenes by the bioprotective L. sakei CTC494 was modelled 163 following the mathematical approach proposed by Costa et al. (2019). This consists of (i) 164 the estimation of the growth parameters lag phase, maximum specific growth rate and 165 maximum population density of L. sakei CTC494 and L. monocytogenes CTC1034 in 166 mono-culture, (ii) the estimation of the inhibition function parameters of three existing 167 microbial competition interaction models using as input the mono-culture kinetic 168 parameters and (iii) the statistical analysis of the performance of the tested models to 169 describe experimental observations.

\subsubsection{Primary growth kinetic parameters and secondary model}


171 The observed growth data for mono-cultured L. sakei CTC494 and L. monocytogenes

172 CTC1034 on hot-smoked sea bream obtained at constant storage temperature $\left(5^{\circ} \mathrm{C}\right)$ were used to fit the primary growth model of Baranyi and Roberts (1994) and the kinetic parameters lag phase $(\lambda, \mathrm{h})$, maximum specific growth rate $\left(\mu_{\max }, 1 / \mathrm{h}\right)$ and maximum population density $\left(N_{\max }, \log \mathrm{CFU} / \mathrm{g}\right)$ were determined for each microorganism. The model was fitted to the experimental data using the program DMFit for Excel v.3.5 (IFR,

\section{Norwich, UK).}

Growth rate at different temperatures was predicted using the secondary model for $\mu_{\max }$ as a function of temperature published by Costa et al. (2019). This model was developed

180 in fish juice of sea bream for the same bacterial strains used in this study and was validated on fresh filleted sea bream stored under isothermal and non-isothermal conditions. The model corresponded to a square root type equation (Ratkowsky et al., 1982) with parameter values $b$ and $T_{\min }$ being 0.028 and $-4.5^{\circ} \mathrm{C}$, respectively for L. sakei CTC494, and 0.026 and $-3.4{ }^{\circ} \mathrm{C}$, respectively for L. monocytogenes CTC1034. Since an effect of the fish matrix and vacuum conditions on $\mu_{\max }$ was expected, an adjustment factor was applied to simulate the microbial interaction on smoked sea bream. The adjustment factor for $\mu_{\max }$ of each microorganism was calculated as the ratio between the $\mu_{\max }$ values obtained in this study from mono-culture experiments at $5{ }^{\circ} \mathrm{C}$ in smoked sea bream and in fish juice by Costa et al. (2019).

\subsubsection{Microbial interaction models}

191 Three existing microbial competition models were evaluated to assess their ability to 192 describe the growth response of the bioprotective effect of $L$. sakei CTC494 on $L$. 193 monocytogenes at isothermal and non-isothermal storage conditions: the Jameson effect 194 model, a modified version of the Jameson effect model and the Lotka-Volterra model. 
195 The generic system of equations (Eq. 1) of the tested interaction models, applied in their

196 implicit form to co-cultured L. sakei CTC494 (subscript '1') and L. monocytogenes

197 (subscript '2'), can be expressed as:

198

$199 \quad\left[\frac{d N_{1}(t)}{d t}=N_{1} \cdot \mu_{\max 1} \cdot\left(\frac{Q_{1}}{1+Q_{1}}\right) \cdot f_{1}(t)\right.$

200

$\frac{d N_{2}(t)}{d t}=N_{2} \cdot \mu_{\max 2} \cdot\left(\frac{Q_{2}}{1+Q_{2}}\right) \cdot f_{2}(t)$

$201\left\{\frac{d Q_{1}}{d t}=Q_{1-1} \cdot \mu_{\max 1}\right.$

202

203

$Q_{0}=\frac{1}{e^{\left(\mu_{\max } \cdot \lambda\right)}-1}$

204 where $N$ is the cell concentration $(\mathrm{CFU} / \mathrm{g})$ at time $t, \mu_{\max }$ is the maximum specific growth 205 rate (1/h), $Q$ and $Q_{0}$ is a measure of the physiological state of cells at time $t$ and $t=0$, 206 respectively, $f(\mathrm{t})$ is an inhibition function and $\lambda(\mathrm{h})$ the lag phase duration of the cells.

207 The different microbial competition models were tested based on the generic system of 208 equations in Eq. (1) and using the corresponding inhibition function $f(\mathrm{t})$ described in 209 either Eq. (2), or (3) or (4) for each type of model.

210 The empirical Jameson effect model is based on the assumption that all bacterial 211 populations stop growing when the dominant culture reaches its maximum population 212 density (Giménez and Dalgaard, 2004). In this case, the inhibition function $f(\mathrm{t})$ is defined 213 as:

$214\left\{\begin{array}{l}f_{1}(t)=\left(1-\frac{N_{1}}{N_{\max 1}}\right) \cdot\left(1-\frac{N_{2}}{N_{\max 2}}\right) \\ f_{2}(t)=\left(1-\frac{N_{2}}{N_{\max 2}}\right) \cdot\left(1-\frac{N_{1}}{N_{\max 1}}\right)\end{array}\right.$ 
216 where $N_{\max }$ is the maximum cell density (CFU/g) for each population with subscript ' 1 ' 217 and '2' for L. sakei CTC494 and L. monocytogenes, respectively, and other parameters 218 are as indicated in Eq. (1).

219 The modified Jameson effect model was based on a modification of the inhibition 220 function $f_{2}(\mathrm{t})$ in Eq. (2), in which the maximum density of population 1 (i.e. L. sakei 221 CTC494; $N_{\max 1}$ ) is replaced by a critical population density, being typically lower than its $222 N_{\max 1}$ (Cornu et al., 2011; Le Marc et al., 2009). This parameter describes the 223 concentration value of the population 1 that results in the stop of the growth of population

224 2. This critical concentration value can be related to the production of an inhibitory 225 substance, at a certain level, able to inhibit growth of the other population. In addition, if $226 N_{C P D 1}<N_{1}<N_{\max 1}$, then $f_{2}(\mathrm{t})<0$, which describes a decline of population 2.

227

$f_{2}(t)=\left(1-\frac{N_{2}}{N_{\max 2}}\right) \cdot\left(1-\frac{N_{1}}{N_{C P D 1}}\right)$

where $N_{C P D 1}$ is the critical population density of population 1 (i.e. L. sakei CTC494; $\mathrm{CFU} / \mathrm{g}$ ) and other parameters are as indicated in Eqs. (1) and (2).

230 Finally, it was used the classical predator-prey (Lotka-Volterra) model for interspecific 231 bacterial competition (Dens et al., 1999; Powell et al., 2004), which includes two 232 coefficients of interaction ( $\alpha_{12}$ and $\left.\alpha_{21}\right)$ measuring the competitive effect of species 1 on 233 species 2 and vice-versa. In our study, the competition term of $L$. monocytogenes on $L$. 234 sakei $\left(\alpha_{21}\right)$ was fixed to zero since we assumed that the pathogen did not influence growth 235 of L. sakei CTC494 due to the higher concentration, shorter lag time and faster growth of 236 the LAB strain (Mejlholm and Dalgaard, 2015; Møller et al., 2013). Therefore, the tested 237 model includes one inhibition function $f_{1}(\mathrm{t})$ formulated as:

$238 f_{1}(t)=\left(1-\frac{N_{1}+\alpha_{12} \cdot N_{2}}{N_{\max 1}}\right)$ 
239 where $\alpha_{12}$ is the competition term of L. sakei CTC494 on L. monocytogenes and other 240 parameters are as indicated in Eqs. (1) and (2).

241 Simultaneous growth of L. sakei CTC494 and L. monocytogenes was described by

242 estimating the inhibition parameter for each tested model, using as input the kinetic 243 parameters estimated by the Baranyi and Roberts primary model from mono-culture data $244\left(\lambda, N_{\max }\right)$, together with the square root function for $\mu_{\max }$ reported by Costa et al. (2019) to 245 account for the temperature effect. The parameter estimations were performed in 246 Microsoft Excel with Solver add-in tool using numerical integration with a time step of $2470.5 \mathrm{~h}$ (Microsoft Corp., Redmond, WA, USA). The interaction factors $\left(C P D_{1}, \alpha_{12}\right)$ were 248 estimated by regression analysis (MS Excel) using kinetic parameters from mono-culture 249 experiments and the secondary model by Costa et al. (2019).

\subsubsection{Interaction models prediction performance}

251 The capacity of the prediction of the tested models under constant and dynamic 252 temperature storage conditions was assessed by the statistical indexes Root Mean Squared 253 Error (RMSE) and corrected Akaike Information Criterion (AICc) calculated as shown in 254 Eqs. (5) and (6), respectively.

$255 R M S E=\sqrt{\frac{\sum(\text { fitted-observed })^{2}}{n-p}}$

$256 \quad A I C C=n \cdot \ln \left(\frac{R S S}{n}\right)+2 \cdot(p+1)+\frac{2 \cdot(p+1) \cdot(p+2)}{n-p-2}$

257 where $n$ is the number of observations and $p$ is the number of model parameters to be 258 estimated in Eqs. (5) and (6) and RSS is the residual sum of squares in Eq. (6).

259 In addition to the statistical performance described above, the Acceptable Simulation 260 Zone (ASZ) method was used to evaluate the prediction capacity of the interaction models 
261 at dynamic storage temperatures. The acceptable interval was defined as $\pm 0.5 \log$-units 262 from the simulated growth of L. sakei or L. monocytogenes. The simulations were 263 considered acceptable when at least $70 \%$ of the observed counts were within the ASZ 264 (Oscar, 2005).

265

266 


\section{RESULTS AND DISCUSSION}

268

269

\subsection{Sensory evaluation of the bioprotective L. sakei CTC494 on smoked sea bream}

270 The average sensory scores and sensory deterioration rates obtained for the three evaluated attributes during storage at $5{ }^{\circ} \mathrm{C}$ for non-inoculated (control) and inoculated samples are showed in Fig. 1 and Table 1, respectively. Overall, no significant differences $(p>0.05)$ were found between the spoilage rates obtained for control and inoculated samples with 2 and $4 \log$ CFU/g of L. sakei CTC494 throughout the storage period, with sensory scores denoting, in general, "very good" or "good" quality (4-9). On the contrary, sensory scores were < 4 at 11 days for samples inoculated with L. sakei CTC494 at $6 \log$ CFU/g for the attribute appearance (Fig. 1A). Moreover, spoilage rates were significantly higher $(\mathrm{p} \leq 0.05)$ at inoculation level of $6 \log \mathrm{CFU} / \mathrm{g}$, indicating that such a high inoculation level may cause undesired changes to the hot-smoked sea bream as compared to the samples of lower inoculum sizes. Although the smoked fish product was not tested for its taste, it can be assumed that the product was not significantly acidified due to the addition of the LAB strain at levels $\leq 4 \log \mathrm{CFU} / \mathrm{g}$, as indicated by the recorded $\mathrm{pH}$ values at the end of the storage period for control and inoculated samples at $4 \log$ CFU/g L. sakei, which corresponded to $6.0 \pm 0.1$ and $5.9 \pm 0.1$, respectively.

Our results are consistent with those reported by Costa et al. (2019), who also sensory validated the application of L. sakei CTC494 on fresh fish fillets under modified atmosphere packaging (MAP) and found that at levels of 2 and $4 \log$ CFU/g the sensory characteristics were statistically similar to the non-inoculated product. Based on these results, the bioprotection strategy against the pathogen was established in this study by inoculating the product with $4 \log$ CFU/g of L. sakei CTC494 at the beginning of the 291 experiments. 
292 3.2. Evaluating the bioprotective effect of $\mathrm{L}$. sakei CTC494 against $\mathrm{L}$. monocytogenes

293

294

295

296

297

298

299

300

301

302

303

304

305

306

307

308

309

310

311

312

313

314

315

\subsubsection{Mono-culture growth on hot-smoked sea bream}

Regarding the microbiological analysis of endogenous microbiota of hot-smoked sea bream, the concentration of endogenous LAB and MVC obtained at the starting day for control samples were below the detection level $(<1 \log$ CFU/g) and remained below this level for $\mathrm{LAB}$ and $<2 \log \mathrm{CFU} / \mathrm{g}$ for MVC during the evaluated storage period at both constant and dynamic temperature conditions (data not shown). In addition, $L$. monocytogenes was not detected in any control sample. The initial $\mathrm{a}_{\mathrm{w}}$ and $\mathrm{pH}$ of noninoculated (control) samples corresponded to $0.96 \pm 0.007$ and $6.0 \pm 0.1$, respectively.

The kinetic parameters and their standard errors estimated by the Baranyi and Roberts model for experiments in mono-cultures at $5{ }^{\circ} \mathrm{C}$ are showed in Table 2. A long lag phase $\lambda$ and an exponential growth rate $\mu_{\max }$ was distinguished for each studied microorganism. L. sakei CTC494 reached the stationary phase after approximately 13 days of storage. However, no stationary phase was observed for L. monocytogenes CTC1034 at the end of the experimental period (20 days), thus the Baranyi and Roberts model (no asymptote) was used to describe the growth behaviour of L. monocytogenes on hot-smoked sea bream. Despite this, the growth potential $(\delta \log )$ of $L$. monocytogenes was $3.5 \log$ during the storage period, which demonstrates that refrigeration at $5{ }^{\circ} \mathrm{C}$ does not prevent the pathogen growth on the studied fish product. L. monocytogenes growth was also observed in other vacuum-packed hot-smoked fish products at refrigeration temperatures (Branciari et al., 2016; Mahmoud et al., 2012; Tosun and Özden, 2014).

The parameter $\mu_{\max }$ in mono-culture experiments was much higher for L. sakei $(0.0621 / \mathrm{h})$ than for L. monocytogenes $(0.0241 / \mathrm{h})$. The $\mu_{\max }$ value obtained for the latter microorganism was lower than that estimated by Costa et al. (2019) for the same strain in 
316 mono-culture in fish juice at $5{ }^{\circ} \mathrm{C}(0.04771 / \mathrm{h})$, corresponding to a reduction of $50 \%$ with

317 respect to the value reported by Costa et al. (2019). These authors found that the $\mu_{\max }$

318 value of L. monocytogenes in the fish product (i.e. MAP fresh filleted sea bream)

319 presented a reduction of $68 \%$ with respect to that observed in fish juice. Differences in

320 reduction rate could be due to the specific characteristics of the product studied herein. In

321 the work by Costa et al. (2019), the presence of indigenous microbiota in fresh fish fillets

322 that was higher can explain a more reduced pathogen growth when compared to smoked

323 sea bream in our study, where MVC and LAB were in low numbers as a consequence of

324 the hot smoking process. In addition, the presence of $\mathrm{CO}_{2}$ in MAP fish fillets has been

325 described as an inhibiting factor influencing L. monocytogenes growth (Bolívar et al.,

326 2018; Provincial et al., 2013). Regarding this, Tosun and Özden (2014) reported that MAP

327 was most effective in controlling the growth of L. monocytogenes in hot-smoked rainbow

328 trout fish fillets at $2{ }^{\circ} \mathrm{C}$ while vacuum packaging had no effect.

329

\subsubsection{Co-culture growth on smoked sea bream}

330 Growth curves of co-cultured L. sakei CTC494 and L. monocytogenes CTC1034 on 331 smoked sea bream are showed in Fig. 2. The LAB strain exhibited a similar lag phase at $3325{ }^{\circ} \mathrm{C}$ and under dynamic temperature profile 1 (around 5 days), which also corresponded 333 to that estimated in mono-culture (Table 2). L. sakei CTC494 showed a shorter lag phase 334 under profile 2, probably due to the abrupt temperature rise at 4 storage days. In addition, 335 the microorganism reached similar $N_{\max }$ values (ca. $8.6 \log \mathrm{CFU} / \mathrm{g}$ ) at all storage 336 temperatures and both (mono and co-) culture conditions. These results suggest, as 337 expected, that the growth of L. sakei CTC494 in co-culture was not affected by the 338 presence of L. monocytogenes. 
339 On the contrary, L. sakei CTC494 significantly reduced the capability of $L$.

340 monocytogenes to grow at the tested inoculation ratio, although its effectiveness was

341 temperature dependent. In this regard, L. sakei CTC494 limited the growth of the

342 pathogen under storage at $5^{\circ} \mathrm{C}(<1 \log$ increase $)$ and at dynamic profile $2(<2 \log$

343 increase) throughout the evaluated period (Figs. 2A and 2C, respectively). Besides, under

344 storage at dynamic profile 1 (Fig. 2B), the growth of L. monocytogenes was inhibited (<

$3450.5 \log$ increase).

346 The competition pattern was similar for all temperature conditions, showcasing a slight

347 L. monocytogenes increase, which ceased when the L. sakei population approached to its

$348 N_{\max }(>8 \log \mathrm{CFU} / \mathrm{g})$. This phenomenon would reflect a non-specific interaction

349 described by a potential Jameson effect between populations. At $5{ }^{\circ} \mathrm{C}$ and profile $1, L$.

350 monocytogenes population showed a remarkable decline after $L$. sakei had reached its

$351 N_{\max }$, but at profile 2 growth of the pathogen slightly continued.

352 The efficacy of $L$. sakei CTC494 against $L$. monocytogenes has already been proved on

353 fish. Aymerich et al. (2019) reported that the growth of L. monocytogenes on three types

354 of cold-smoked salmon inoculated with L. sakei CTC494 was completely inhibited after

35521 days at $8{ }^{\circ} \mathrm{C}$ under vacuum packaging. In other study, the increase of the pathogen was

356 less than $1 \log$ units on fresh filleted sea bream in the presence of the LAB strain after

357 storage under MAP at isothermal and non-isothermal storage conditions (Costa et al.,

358 2019). The degree of inhibition of L. monocytogenes exerted by L. sakei CTC494 in

359 different types of fish products illustrates the capacity of this bioprotective culture as a

360 potential antimicrobial agent (AMA) to be used as part of the L. monocytogenes control

361 alternatives for RTE food defined by the US Food Safety Inspection Service Listeria rule

362 (FSIS, 2014). 


\subsection{Microbial interaction on smoked sea bream}

365 The three microbial interaction models were fitted to the co-culture experimental results 366 with Eq. (1) estimating the corresponding inhibition function parameters (Eq. (2), (3) or 367 (4)). The fitted inhibition parameters $\left(C P D_{1}\right.$ and $\left.\alpha_{12}\right)$ and the goodness-of-fit indexes are 368 presented in Table 3, together with the ASZ values (\%) obtained from the prediction 369 capacity evaluation for dynamic storage temperatures.

To consider the effect of food matrix on $\mu_{\max }$ on the growth simulation at dynamic temperature conditions, the $\mu_{\max }$ for $L$. monocytogenes estimated from the secondary model (based on fish juice) was adjusted by applying an adjustment factor of 0.50 (section 3.2.1) in the tested microbial interaction models. For L. monocytogenes, the LotkaVolterra model showed, in general, the best fitting according to the statistical indices RMSE and AICc, with values varying from 0.334 to 0.536 and -12.19 to -4.65 , respectively. The modified Jameson effect model presented better fitting than the Jameson effect model, except for dynamic profile 2 in which the lowest RMSE value was

For L. sakei CTC494, the three interaction models provided similar fitting, with RMSE values being slightly lower for the Jameson effect model (Table 3). For all tested models, the worst fitting was obtained for profile 2 as indicated by higher RMSE and AICc values (Table 3). In profile 2, it can be also observed that the bioprotector strain exhibited an adaptation delay after the initial lag phase (Fig. 2C). This phenomenon has been previously described as intermediate lag periods induced by abrupt temperature shifts in which cells need to adjust to the changing temperature environment (Swinnen et al., 2005). Intermediate lag periods of significant duration are expected to be present when 
387 the temperature profiles are below the optimal temperature of growth, thus the predictive 388 ability of the secondary models is affected negatively (Longhi et al., 2013). This 389 phenomenon has been previously observed for the same microorganism in fresh fish 390 (Costa et al., 2019) and also for different Lactobacillus strains in other food products 391 stored at dynamic temperatures (Longhi et al., 2013; Silva et al., 2017).

392 Owing to the LAB strain used in our study is a sakacin K producing species, a specific antagonistic effect would be expected due to this potentially inhibitory substance on $L$. monocytogenes. Although the bacteriocin was not quantified to confirm this effect, the fish product and storage temperatures under study might constitute a suitable bacteriocin production-supporting environment. In this regard, Hugas et al. (1998) quantified sakacin K produced by L. sakei CTC494 in MRS broth and observed bacteriocin activity at initial $\mathrm{pH}$ of 6.0 and 6.5 and higher production at low temperatures $\left(4,10\right.$ and $\left.15^{\circ} \mathrm{C}\right)$ compared to abuse storage $\left(20,25\right.$ and $\left.30^{\circ} \mathrm{C}\right)$. In addition, Leroy and De Vuyst (2001) suggested that extremely rich environments (e.g. MRS broth) will not necessarily increase specific bacteriocin production compared to nutrient-depleted environments, as in the present work (i.e. sugar limitation). Apparently, bacteriocin production is stimulated by less favorable growth conditions, such as low temperatures and competing microbiota (Delboni and Yang, 2017).

405 Regarding the prediction capacity of the tested models under dynamic profile 1, all 406 models were able to describe satisfactory the simultaneous microbial growth, with $75 \%$ 407 of the observations within the ASZ, excepting for the Jameson model, for which the value 408 for L. monocytogenes was 37.5\%. Under dynamic profile 2, none of the models provided 409 reasonable predictions for co-cultured L. sakei CTC494 $(<70 \%)$. This lack of prediction 410 could be explained by the existence of an intermediate lag phase as mentioned in above 
411 lines. However, all models represented adequately the growth response of $L$.

412 monocytogenes, as confirmed by ASZ values equal to $87.5 \%$.

413 The estimated value of the $C P D_{1}$ parameter included in the modified Jameson effect

414 model remained similar for the different temperatures (Table 3). The value of the 415 competition factor of $L$. sakei CTC494 on L. monocytogenes $\left(\alpha_{12}\right)$ estimated by the Lotka416 Volterra model was different depending on the temperature conditions. At $5{ }^{\circ} \mathrm{C}$ and profile 417 1, the obtained $\alpha_{12}$ were above 1 , which properly describes the observed $L$. monocytogenes 418 population decline when $L$. sakei CTC494 reached its $N_{\max }$. In contrast, at profile 2, $\alpha_{12}$ 419 was $<1$, which means that L. monocytogenes growth was slowed down as L. sakei 420 CTC494 approached to its $N_{\max }$, as can be seen in Fig. 2C. This reflects the different effect 421 of non-isothermal conditions on the interaction between both microorganisms. The Lotka-Volterra's coefficient of interaction $(\alpha)$ of different microbial species in diverse food matrices has also been reported in the literature as a temperature-dependent 424 parameter. Some examples are the values of $\alpha$ estimated at different temperatures for the growth of $L$. monocytogenes in fish juice ( $\alpha$-average $=1.4$ between 2.2 and $5{ }^{\circ} \mathrm{C}$ and $\alpha$ average $=1.6$ and 1.8 at 8 and $12{ }^{\circ} \mathrm{C}$, respectively) (Costa et al., 2019), or the polynomial model developed by Møller et al. (2013) which described the effect of temperature on $\alpha$ of the natural microbiota on growth of Salmonella spp. in ground pork during storage between $9{ }^{\circ} \mathrm{C}$ to $24^{\circ} \mathrm{C}$.

430 Most of the studies dealing with microbial interaction responses in fish products have 431 been performed on mixed cultures based on a pathogen and non-bacteriocinogenic 432 microorganisms, being either endogenous or artificially inoculated (Giménez and 433 Dalgaard, 2004; Koseki et al., 2011; Mejlholm and Dalgaard, 2015, 2007). These studies 434 aimed to describe the maximum population density of the pathogen, for which the 435 classical Jameson effect model is used. Other studies have used the Lotka-Volterra model 
436 as in the case of the work by Giuffrida et al. (2007) that analysed the interaction between

437 Aeromonas hydrophila and aerobic natural microbiota on sea bream surfaces considering

438 the complexity of fluctuating environmental conditions and the interspecific bacterial

439 interactions.

$440 \quad 4$ CONCLUSIONS

441 The results from this study contribute to extend the application of the bacteriocinogenic

442 strain L. sakei CTC494 for controlling growth of L. monocytogenes in hot-smoked fish

443 products from Mediterranean aquaculture during refrigerated storage. Results from the

444 challenge tests demonstrated the potential of L. sakei CTC494 applied at a dose of $4 \log$

$445 \mathrm{CFU} / \mathrm{g}$ to limit or inhibit the growth of L. monocytogenes on hot-smoked sea bream under

446 different storage temperatures. Nevertheless, further research is still needed to confirm

447 this inhibitory effect under other possible scenarios such as different temperature profiles

448 (e.g. abrupt temperature changes and abuse temperatures) and process parameters as well

449 as considering batch variability. The proposed modelling approach, based on a validation

450 process in food and the application of adjustment factors to kinetic parameters from the

451 modified Jameson and Lotka-Volterra models, was able to satisfactorily describe the

452 bioprotective effect of $L$. sakei CTC494 on L. monocytogenes in the target fish product.

453 This predictive tool could support the assessment and establishment of bioprotective

454 culture-based strategies aimed at reducing the risk of listeriosis linked to the consumption

455 of RTE hot-smoked sea bream. 


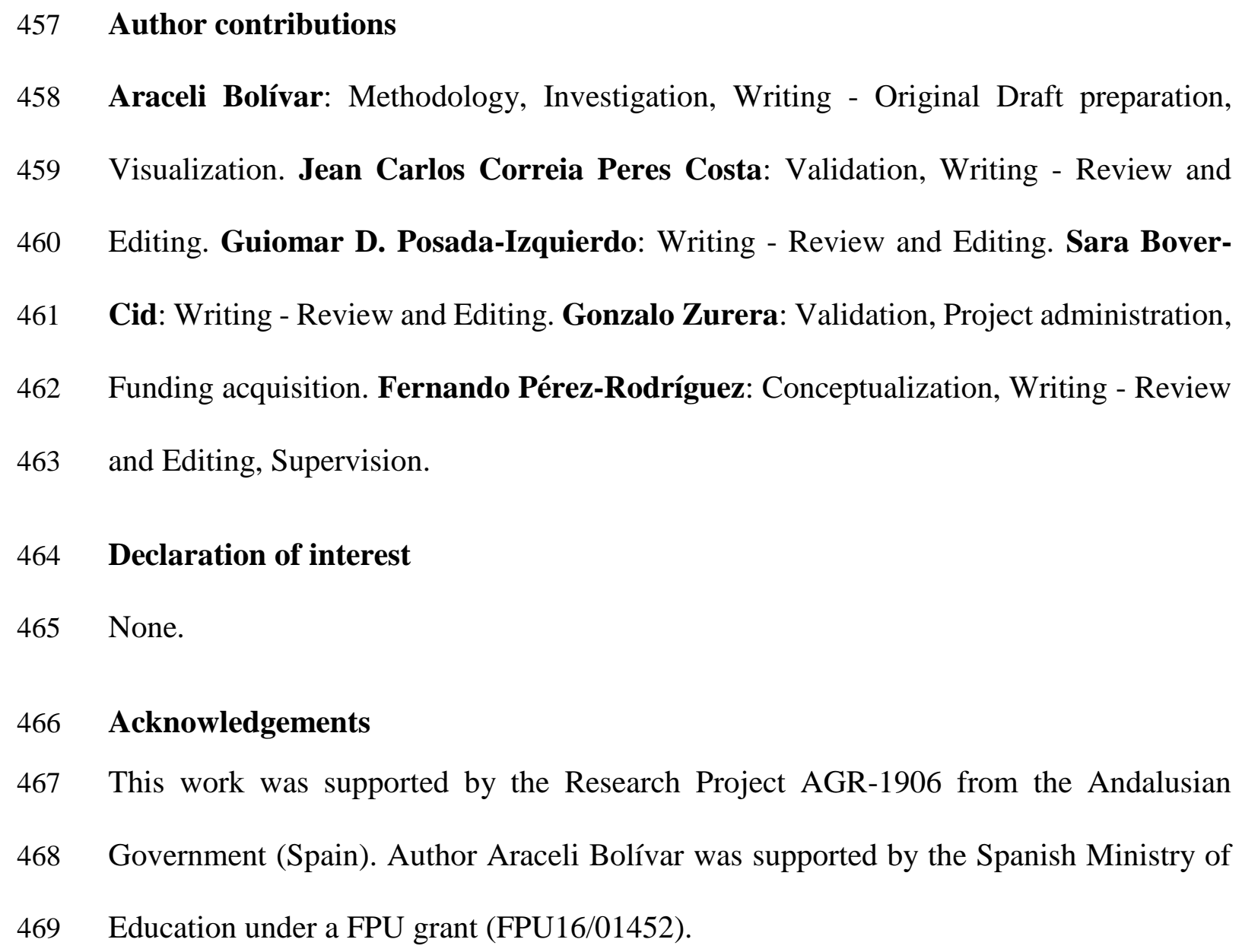

458 Araceli Bolívar: Methodology, Investigation, Writing - Original Draft preparation,

459 Visualization. Jean Carlos Correia Peres Costa: Validation, Writing - Review and

460 Editing. Guiomar D. Posada-Izquierdo: Writing - Review and Editing. Sara Bover-

461 Cid: Writing - Review and Editing. Gonzalo Zurera: Validation, Project administration,

462 Funding acquisition. Fernando Pérez-Rodríguez: Conceptualization, Writing - Review

463 and Editing, Supervision.

\section{Declaration of interest}

465 None.

466 Acknowledgements

467 This work was supported by the Research Project AGR-1906 from the Andalusian 468 Government (Spain). Author Araceli Bolívar was supported by the Spanish Ministry of 469 Education under a FPU grant (FPU16/01452).

\section{0}




\section{REFERENCES}

Aymerich, T., Rodríguez, M., Garriga, M., Bover-Cid, S., 2019. Assessment of the bioprotective potential of lactic acid bacteria against Listeria monocytogenes on vacuum-packed cold-smoked salmon stored at $8^{\circ} \mathrm{C}$. Food Microbiol. 83, 64-70. https://doi.org/10.1016/j.fm.2019.04.011

Baranyi, J., Roberts, T.A., 1994. A dynamic approach to predicting bacterial growth in food. Int. J. Food Microbiol. https://doi.org/10.1016/0168-1605(94)90157-0

Bolívar, A., Costa, J.C.C.P., Posada-Izquierdo, G.D., Valero, A., Zurera, G., PérezRodríguez, F., 2018. Modelling the growth of Listeria monocytogenes in Mediterranean fish species from aquaculture production. Int. J. Food Microbiol. 270. https://doi.org/10.1016/j.ijfoodmicro.2018.02.005

Branciari, R., Valiani, A., Franceschini, R., Ranucci, D., Lupattelli, A., Urbani, E., Ortenzi, R., 2016. Thermal inactivation and growth potential of Listeria monocytogenes in smoked tench. Ital. J. Food Saf. 5. https://doi.org/10.4081/ijfs.2016.5974

Cornu, M., Billoir, E., Bergis, H., Beaufort, A., Zuliani, V., 2011. Modeling microbial competition in food: Application to the behavior of Listeria monocytogenes and lactic acid flora in pork meat products. Food Microbiol. 28, 639-647. https://doi.org/10.1016/j.fm.2010.08.007

Costa, J.C.C.P., Bover-Cid, S., Bolívar, A., Zurera, G., Pérez-Rodríguez, F., 2019. Modelling the interaction of the sakacin-producing Lactobacillus sakei CTC494 and Listeria monocytogenes in filleted gilthead sea bream (Sparus aurata) under modified atmosphere packaging at isothermal and non-isothermal conditions. Int. J. Food Microbiol. 297, 72-84. https://doi.org/10.1016/j.ijfoodmicro.2019.03.002

Delboni, R.R., Yang, H.M., 2017. Mathematical model of interaction between bacteriocin-producing lactic acid bacteria and Listeria. Part 2: bifurcations and applications. Bull. Math. Biol. 79, 2273-2301. https://doi.org/10.1007/s11538-0170330-1

Dens, E.J., Vereecken, K.M., Van Impe, J.F., 1999. A prototype model structure for mixed microbial populations in homogeneous food products. J. Theor. Biol. 201, 159-170. https://doi.org/10.1006/jtbi.1999.1021

EFSA (European Food Safety Authority), 2018. Update of the list of QPSrecommended biological agents intentionally added to food or feed as notified to EFSA 8: suitability of taxonomic units notified to EFSA until March 2018. EFSA J. 2018, 16:5315. https://doi.org/10.2903/j.efsa.2018.5315

EFSA BIOHAZ Panel (EFSA Panel on Biological Hazards), 2018. Scientific Opinion on the Listeria monocytogenes contamination of ready-to-eat foods and the risk for human health in the EU. EFSA J. 2018, 16:5134. https://doi.org/10.2903/j.efsa.2018.5134

FSIS, 2014. Compliance guideline: controlling Listeria monocytogenes in post-lethality exposed ready-to-eat meat and poultry products. Food Safety and Inspection Service. U.S. Department of Agriculture, Washington, pp. 143. https://www.fsis.usda.gov/wps/wcm/connect/d3373299-50e6-47d6-a577- 

January 2020.

Garriga, M., Aymerich, M.T., Costa, S., Monfort, J.M., Hugas, M., 2002. Bactericidal synergism through bacteriocins and high pressure in a meat model system during storage. Food Microbiol. 19, 509-518.

Ghanbari, M., Jami, M., Domig, K.J., Kneifel, W., 2013. Seafood biopreservation by lactic acid bacteria - A review. LWT - Food Sci. Technol. https://doi.org/10.1016/j.lwt.2013.05.039

Giménez, B., Dalgaard, P., 2004. Modelling and predicting the simultaneous growth of Listeria monocytogenes and spoilage micro-organisms in cold-smoked salmon. J. Appl. Microbiol. 96, 96-109. https://doi.org/10.1046/j.1365-2672.2003.02137.x

Giuffrida, A., Ziino, G., Valenti, D., Donato, G., Panebianco, A., 2007. Application of an interspecific competition model to predict the growth of Aeromonas hydrophila on fish surfaces during refrigerated storage. Arch. Lebensmittelhyg. 58, 136-141. https://doi.org/10.2377/0003-925x-58-136

Hugas, M., Pages, F., Garriga, M., Monfort'the, J.M., 1998. Application of the bacteriocinogenic Lactobacillus sakei CTC494 to prevent growth of Listeria in fresh and cooked meat products packed with different atmospheres. Food Microbiol. 15, 639-650.

Hwang, C.-A., 2007. Effect of salt, smoke compound, and storage temperature on the growth of Listeria monocytogenes in simulated smoked salmon. J. Food Prot. 70, 2321-2328.

Jameson, J. E., 1962. A discussion of the dynamics of Salmonella enrichment. J. Hyg. 60, 193-207. https://doi.org/10.1017/s0022172400039462

Koseki, S., Takizawa, Y., Miya, S., Takahashi, H., Kimura, B., 2011. Modeling and predicting the simultaneous growth of Listeria monocytogenes and natural flora in minced tuna. J. Food Prot. 74, 176-187. https://doi.org/10.4315/0362-028x.jfp-10258

Le Marc, Y., Valík, L., Medved'ová, A., 2009. Modelling the effect of the starter culture on the growth of Staphylococcus aureus in milk. Int. J. Food Microbiol. 129, 306311. https://doi.org/10.1016/j.ijfoodmicro.2008.12.015

Leistner, L., 2000. Basic aspects of food preservation by hurdle technology. Int. J. Food Microbiol. 55, 181-186. https://doi.org/10.1016/S0168-1605(00)00161-6

Leroi, F., Cornet, J., Chevalier, F., Cardinal, M., Coeuret, G., Chaillou, S., Joffraud, J.J., 2015. Selection of bioprotective cultures for preventing cold-smoked salmon spoilage. Int. J. Food Microbiol. 213, 79-87. https://doi.org/10.1016/j.ijfoodmicro.2015.05.005

Leroy, F., De Vuyst, L., 2001. Growth of the bacteriocin-producing Lactobacillus sakei strain CTC 494 in MRS broth is strongly reduced due to nutrient exhaustion: A nutrient depletion model for the growth of lactic acid bacteria. Appl. Environ. Microbiol. 67, 4407-4413. https://doi.org/10.1128/AEM.67.10.4407-4413.2001 
Longhi, D.A., Dalcanton, F., Aragão, G.M.F. de, Carciofi, B.A.M., Laurindo, J.B., 2013. Assessing the prediction ability of different mathematical models for the growth of Lactobacillus plantarum under non-isothermal conditions. J. Theor. Biol. 335, 88-96. https://doi.org/10.1016/j.jtbi.2013.06.030

Mahmoud, B.S.M., Coker, R., Su, Y.C., 2012. Reduction in Listeria monocytogenes and spoilage bacteria on smoked catfish using X-ray treatments. Lett. Appl. Microbiol. 54, 524-529. https://doi.org/10.1111/j.1472-765X.2012.03242.X

Mejlholm, O., Boknaes, N., Dalgaard, P., 2015. Development and validation of a stochastic model for potential growth of Listeria monocytogenes in naturally contaminated lightly preserved seafood. Food Microbiol. 45, 276-289. https://doi.org/10.1016/j.fm.2014.06.006

Mejlholm, O., Dalgaard, P., 2007. Modeling and predicting the growth of lactic acid bacteria in lightly preserved seafood and their inhibiting effect on Listeria monocytogenes. J. Food Prot. 11, 2485-2497. https://doi.org/10.4315/0362-028x70.11 .2485

Mejlholm, O., Dalgaard, P., 2015. Modelling and predicting the simultaneous growth of Listeria monocytogenes and psychrotolerant lactic acid bacteria in processed seafood and mayonnaise-based seafood salads. Food Microbiol. 46, 1-14. https://doi.org/10.1016/j.fm.2014.07.005

Møller, C.O.A., Ilg, Y., Aabo, S., Christensen, B.B., Dalgaard, P., Hansen, T.B., 2013. Effect of natural microbiota on growth of Salmonella spp. in fresh pork - A predictive microbiology approach. Food Microbiol. 34, 284-295. https://doi.org/10.1016/j.fm.2012.10.010

Oscar, T.P., 2005. Validation of lag time and growth rate models for Salmonella Typhimurium: acceptable prediction zone method. J. Food Sci. 70, M129-M137. https://doi.org/10.1111/j.1365-2621.2005.tb07103.x

Powell, M., Schlosser, W., Ebel, E., 2004. Considering the complexity of microbial community dynamics in food safety risk assessment. Int. J. Food Microbiol. 90, 171-179. https://doi.org/10.1016/S0168-1605(03)00106-5

Provincial, L., Guillen, E., Gil, M., Alonso, V., Roncales, P., Beltran, J.A., 2013. Survival of Listeria monocytogenes and Salmonella Enteritidis in sea bream (Sparus aurata) fillets packaged under enriched $\mathrm{CO}_{2}$ modified atmospheres. Int. J. Food Microbiol. 162, 213-219. https://doi.org/10.1016/j.ijfoodmicro.2013.01.015

Ratkowsky, D., Olley, J., McMeekin, T., 1982. Relationship between temperature and growth rate of bacterial cultures. J. Bacteriol. 149, 1-5.

Said, L. Ben, Ne Gaudreau, H., Dallaire, L., Le Tessier, M., Fliss, I., 2019. Bioprotective culture: a new generation of food additives for the preservation of food quality and safety. Ind. Biotechnol. 15, 138-147. https://doi.org/10.1089/ind.2019.29175.lbs

Silva, N.B. da, Longhi, D.A., Martins, W.F., Laurindo, J.B., Aragão, G.M.F. de, Carciofi, B.A.M., 2017. Modeling the growth of Lactobacillus viridescens under non-isothermal conditions in vacuum-packed sliced ham. Int. J. Food Microbiol. 240, 97-101. https://doi.org/10.1016/j.ijfoodmicro.2016.05.014 
Swinnen, I.A.M., Bernaerts, K., Gysemans, K., Van Impe, J.F., 2005. Quantifying microbial lag phenomena due to a sudden rise in temperature: A systematic macroscopic study. Int. J. Food Microbiol. 100, 85-96. https://doi.org/10.1016/j.ijfoodmicro.2004.10.022

Tahiri, I., Desbiens, M., Kheadr, E., Lacroix, C., Fliss, I., 2009. Comparison of different application strategies of divergicin M35 for inactivation of Listeria monocytogenes in cold-smoked wild salmon. Food Microbiol. 26, 783-793. https://doi.org/10.1016/j.fm.2009.05.003

Tosun, Ş.Y., Özden, Ö., 2014. Survey of inhibition of Listeria monocytogenes in hotsmoked rainbow trout fillets for food safety. J. Food Process. Preserv. 38, 338346. https://doi.org/10.1111/j.1745-4549.2012.00781.x

Valenti, D., Giuffrida, A., Zinno, G., Giarratana, F., Spagnolo, B., Panebianco, A., 2013. Modelling bacterial dynamics in food products: Role of environmental noise and interspecific competition. J. Mod. Phys. 4, 1059-1065.

Vasilopoulos, C., De Mey, E., Dewulf, L., Paelinck, H., De Smedt, A., Vandendriessche, F., De Vuyst, L., Leroy, F., 2010. Interactions between bacterial isolates from modified-atmosphere-packaged artisan-type cooked ham in view of the development of a bioprotective culture. Food Microbiol. 27, 1086-1094. https://doi.org/10.1016/j.fm.2010.07.013

Yanar, Y., Çelik, M., Akamca, E., 2006. Effects of brine concentration on shelf-life of hot-smoked tilapia (Oreochromis niloticus) stored at $4{ }^{\circ} \mathrm{C}$. Food Chem. 97, 244247. https://doi.org/10.1016/j.foodchem.2005.03.043 

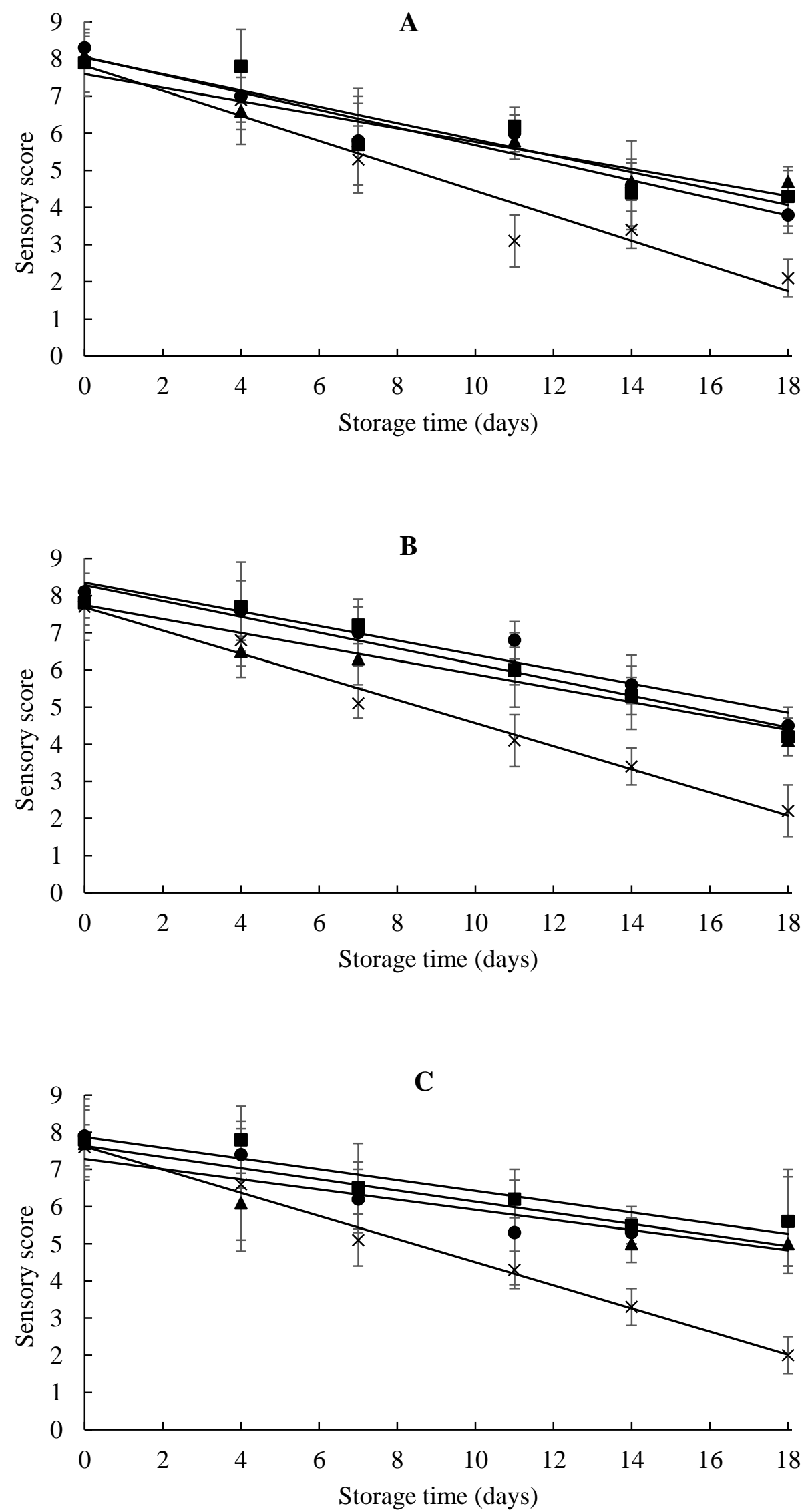

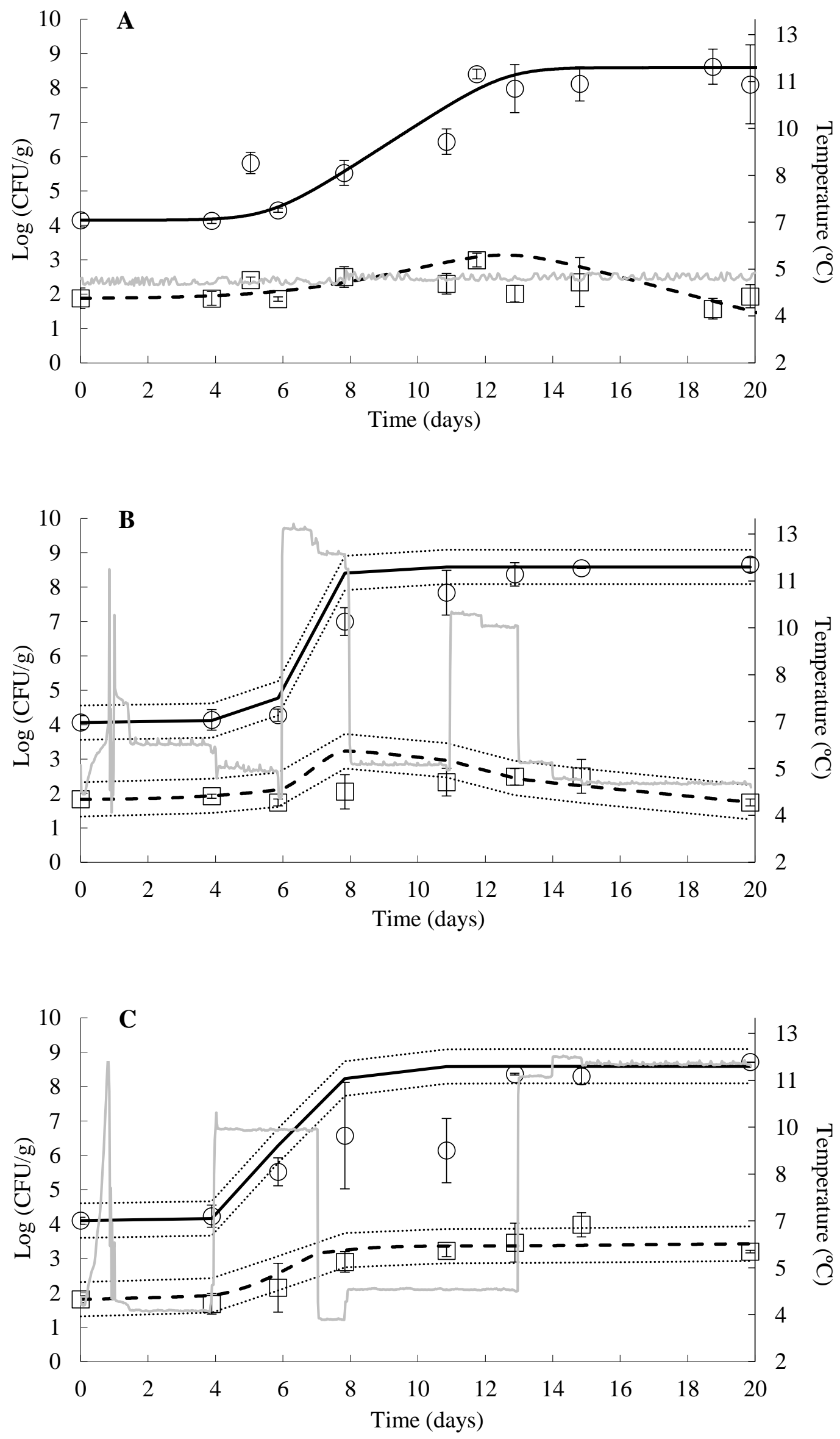
Figure 1. Sensory scores obtained for the attribute appearance (A), odour (B) and texture (C) for vacuum-packed hot-smoked sea bream samples non-inoculated $(\bullet)$ and inoculated at different initial levels of L. sakei CTC494: $2 \log$ CFU/g (•), 4 log CFU/g (ム) and $6 \log$ CFU/g (×) under storage at $5{ }^{\circ} \mathrm{C}$. Data point are the mean values from five panelists with vertical lines denoting \pm the standard deviation.

Figure 2. Experimental growth data of Listeria monocytogenes CTC1034 ( $\square$ ) and Lactobacillus sakei CTC494 (०) on vacuum-packed hot-smoked sea bream during storage at $5{ }^{\circ} \mathrm{C}(\mathrm{A})$ and under dynamic profile 1 (B) and 2 (C). Dashed and solid lines stand for the fit by the Lotka-Volterra model for L. monocytogenes and L. sakei, respectively. Dotted fine lines define the acceptable simulation zone (ASZ) in (B) and (C). The recorded storage temperature is shown as grey solid line. Growth data points are the mean values from two independent trials with vertical lines showing \pm the standard deviation. 


\section{Table 1}

Sensory deterioration rates (slope \pm standard error) obtained for vacuum-packed hot-smoked sea bream samples non-inoculated (control) and inoculated at different initial levels of L. sakei CTC494 (2, 4 and $6 \log \mathrm{CFU} / \mathrm{g})$ under storage at $5^{\circ} \mathrm{C}$. Values for the same attribute with different uppercase letters are significantly different $(\mathrm{p} \leq 0.05)$.

\begin{tabular}{llll}
\hline $\begin{array}{l}\text { Initial level (log } \\
\text { CFU/g) }\end{array}$ & Appearance & Odour & Texture \\
\hline Control & $-0.237 \pm 0.03^{\mathrm{A}}$ & $-0.195 \pm 0.03^{\mathrm{A}}$ & $-0.150 \pm 0.04^{\mathrm{A}}$ \\
2 & $-0.220 \pm 0.05^{\mathrm{A}}$ & $-0.213 \pm 0.03^{\mathrm{A}}$ & $-0.145 \pm 0.03^{\mathrm{A}}$ \\
4 & $-0.182 \pm 0.03^{\mathrm{A}}$ & $-0.186 \pm 0.03^{\mathrm{A}}$ & $-0.136 \pm 0.03^{\mathrm{A}}$ \\
6 & $-0.337 \pm 0.04^{\mathrm{B}}$ & $-0.331 \pm 0.02^{\mathrm{B}}$ & $-0.311 \pm 0.01^{\mathrm{B}}$ \\
\hline
\end{tabular}




\section{Table 2}

Estimated lag time $(\lambda)$, maximum specific growth rate $\left(\mu_{\max }\right)$, maximum population density $\left(N_{\max }\right)$ \pm associated standard error for the individual growth curves of $L$. sakei CTC494 and $L$. monocytogenes CTC1034 on vacuum-packed hot-smoked sea bream at $5{ }^{\circ} \mathrm{C}$.

\begin{tabular}{lllll}
\hline & $\lambda$ (hours) & $\mu_{\max }(1 / \mathrm{h})$ & $N_{\max }(\log \mathrm{CFU} / \mathrm{g})$ & $\operatorname{Adj}^{2, \mathrm{a}}$ \\
\hline L. sakei CTC494 & $133.1 \pm 33.7$ & $0.0620 \pm 0.006$ & $8.59 \pm 0.17$ & 0.977 \\
$\begin{array}{l}\text { L. monocytogenes } \\
\text { CTC1034 }\end{array}$ & $160.3 \pm 39.5$ & $0.0240 \pm 0.001$ & $\mathrm{ne}^{\mathrm{b}}$ & 0.954 \\
\hline
\end{tabular}

${ }^{a}$ Adjusted coefficient of determination of the fitted Baranyi and Roberts model (Adj. $\mathrm{R}^{2}$ ).

${ }^{\mathrm{b}}$ The Baranyi and Roberts model (no asymptote) was fitted to L. monocytogenes growth data (ne). 


\section{Table 3}

Estimated inhibition parameters $\left(C P D_{1}, \alpha_{12}\right)$ and goodness-of-fit of the three microbial interaction models used to describe the bioprotective capacity of $L$. sakei CTC494 against L. monocytogenes CTC1034 on vacuum-packed hot-smoked sea bream stored at different temperatures.

\begin{tabular}{|c|c|c|c|c|c|c|c|c|c|c|c|c|}
\hline \multirow[t]{2}{*}{ Microorganism } & \multirow[t]{2}{*}{ Temp. $\left({ }^{\circ} \mathrm{C}\right)$} & \multirow[t]{2}{*}{$n^{\mathrm{a}}$} & \multicolumn{2}{|c|}{ Jameson model } & \multicolumn{4}{|c|}{ Modified Jameson model } & \multicolumn{4}{|c|}{ Lotka-Volterra model } \\
\hline & & & RMSE $^{b}$ & $\operatorname{ASZ}(\%)^{\mathrm{c}}$ & $C P D_{1}{ }^{d}$ & RMSE & $\mathrm{AICc}^{\mathrm{e}}$ & $\operatorname{ASZ}(\%)$ & $\alpha_{12}^{f}$ & RMSE & $\mathrm{AICc}$ & $\operatorname{ASZ}(\%)$ \\
\hline \multirow[t]{3}{*}{ L. sakei CTC494 } & 5.0 & 11 & 0.611 & - & 8.28 & 0.676 & -1.39 & - & 2.019 & 0.642 & -5.32 & - \\
\hline & Profile $1^{\mathrm{g}}$ & 8 & 0.595 & 75.0 & 8.44 & 0.688 & 3.72 & 75.0 & 1.418 & 0.638 & -1.87 & 75.0 \\
\hline & Profile $2^{\mathrm{h}}$ & 8 & 1.087 & 62.5 & 8.59 & 1.256 & 13.34 & 62.5 & 0.989 & 1.163 & 7.74 & 62.5 \\
\hline \multirow{3}{*}{$\begin{array}{l}\text { L. monocytogenes } \\
\text { CTC1034 }\end{array}$} & 5.0 & 11 & 0.879 & - & - & 0.506 & -7.78 & - & - & 0.480 & -11.71 & - \\
\hline & Profile 1 & 8 & 0.932 & 37.5 & - & 0.579 & 0.95 & 75.0 & - & 0.536 & -4.65 & 75.0 \\
\hline & Profile 2 & 8 & 0.314 & 87.5 & - & 0.361 & -6.59 & 87.5 & - & 0.334 & -12.19 & 87.5 \\
\hline
\end{tabular}

${ }^{\mathrm{a}}$ Number of observations $(n)$.

${ }^{\mathrm{b}}$ Root Mean Squared Error (RMSE).

${ }^{\mathrm{c}}$ Percentage $(\%)$ of observed values falling within the acceptable simulation zone (ASZ), defined as the simulated value \pm 0.5 log-units.

${ }^{\mathrm{d}}$ Critical population density for L. sakei $\left(C P D_{1}, \log \mathrm{CFU} / \mathrm{g}\right)$ estimated by the modified Jameson effect model fitting Eqs. (1) and (3).

${ }^{\text {e }}$ Corrected Akaike Information Criterion (AICc).

${ }^{\mathrm{f}}$ Competition factor of L. sakei on L. monocytogenes $\left(\alpha_{12}\right)$ estimated by the Lotka-Volterra model fitting Eqs. (1) and (4).

${ }^{\mathrm{g}}$ Dynamic temperature profile ranging from 3.6 to $12.8^{\circ} \mathrm{C}$ (average of $6.3^{\circ} \mathrm{C}$ ).

${ }^{\mathrm{h}}$ Dynamic temperature profile ranging from 3.3 to $11.8^{\circ} \mathrm{C}$ (average of $7.6^{\circ} \mathrm{C}$ ). 